PROCEEDINGS OF THE

AMERICAN MATHEMATICAL SOCIETY

Volume 00, Number 0, Pages 000-000

S $0002-9939$ (XX)0000-0

\title{
PERMUTATION MODULES FOR THE SYMMETRIC GROUP
}

\author{
CLARA FRANCHI, ALEXANDER A. IVANOV, AND MARIO MAINARDIS \\ (Communicated by )
}

\begin{abstract}
In this paper we present a general method for computing the irreducible components of the permutation modules of the symmetric groups over a field $F$ of characteristic 0 . We apply this machinery to determine the decomposition into irreducible submodules of the $F\left[S_{n}\right]$-permutation module on the right cosets of the normaliser in $S_{n}$ of the subgroup generated by a permutation of type $(3,3)$.
\end{abstract}

\section{INTRODUCTION AND NOTATIONS}

Throughout this paper, $G$ is a finite group and $F$ a field of characteristic 0 or coprime to $|G|$. For a subgroup $L$ of $G$, denote by $M_{L}^{G}$ the $F[G]$-permutation module associated to the action of $G$ by right multiplication on the set of right cosets of $L$ in $G$. If $S$ is an irreducible $F[G]$-module, the multiplicity of $S$ in $M_{L}^{G}$ is the number of the composition factors of $M_{L}^{G}$ isomorphic to $S$. If $H$ and $N$ are subgroups of $G$, with $H \leq N \leq N_{G}(H)$, let, for every $x \in N$,

$$
x^{\tau}: M_{H}^{G} \rightarrow M_{H}^{G}
$$

be the linear map defined, for every right coset $H g$ of $H$ in $G$, by

$$
(H g)^{x^{\tau}}:=H x^{-1} g \text {. }
$$

Note that, since $N \leq N_{G}(H), x^{\tau}$ is a well defined $F[G]$-isomorphism of $M_{H}^{G}$ and the map

$$
\tau: N \rightarrow \operatorname{Aut}_{F[G]}\left(M_{H}^{G}\right),
$$

defined, for every $x \in N$, by $x \mapsto x^{\tau}$, is a representation of $N$ with kernel $H$. This induces on $M_{H}^{G}$ an $(F[G], F[N])$-bimodule structure, which on turn defines, in the natural way, an $F[N]$-module structure on $\operatorname{Hom}_{F[G]}\left(S, M_{H}^{G}\right)$ for every $F[G]$-module $S$ (see [5, Proposition 3.5]). Clearly $\tau$ depends on the subgroup $H$. When needed, we shall specify this by indexing $\tau$ with the subgroup $H$. In Section 2, we prove

Theorem 1.1. Let $H$ and $N$ be subgroups of $G$ with $H \leq N \leq N_{G}(H)$. Then, for every irreducible $F[G]$-module $S$, the multiplicity of $S$ in $M_{N}^{G}$ is equal to the multiplicity of the trivial $F[N]$-module in $\operatorname{Hom}_{F[G]}\left(S, M_{H}^{G}\right)$.

In Section 2 we prove a variation of Theorem 1.1 which will turn to be useful for simplifying computations in certain situations. In Sections 3 and 4 we apply the above results to give a general method for computing the irreducible submodules of $M_{N}^{S_{n}}$, where $N$ is a subgroup of the symmetric group $S_{n}$ on $\{1, \ldots, n\}$ ( $n$ a positive

2010 Mathematics Subject Classification. Primary 20C30, 20C15.

(C)XXXX American Mathematical Society 
integer). The idea, here, is to trap $N$ between a Young subgroup $Y_{\mu}$, associated to a suitable partition $\mu$, and its normaliser in $S_{n}$ and use the theory of $\lambda$-tableaux of type $\mu$ to describe explicitly the action $\tau$. In Section 5 we apply the above machinery to the case where $\mu=(n-6,1,1,1,1,1,1)$ and prove

Theorem 1.2. Assume the characteristic of $F$ is 0 . Let $N$ be the normalizer in $S_{n}$ of a subgroup generated by a permutation of type $(3,3)$. Then $M_{N}^{S_{n}}$ decomposes as a direct sum of irreducible submodules as follows:

$$
M_{N}^{S_{n}}=\bigoplus_{\lambda \in \Lambda} m_{\lambda} S^{\lambda}
$$

where for every $\lambda \in \Lambda, S^{\lambda}$ is the Specht module associated to $\lambda$, the elements of the set $\Lambda$ are listed in the first column of Table 1 , and, for each $n \geq 6$, the values $m_{\lambda}$ are given in the remaining columns (note that, for small values of $n$, some of the displayed $\lambda$ 's do not correspond to a partition of $n$; boxes corresponding to such pairs $(\lambda, n)$ are left blank).

\begin{tabular}{|l|c|c|c|c|c|c|c|}
\hline$\lambda$ & $n=6$ & $n=7$ & $n=8$ & $n=9$ & $n=10$ & $n=11$ & $n \geq 12$ \\
\hline$(n)$ & 1 & 1 & 1 & 1 & 1 & 1 & 1 \\
\hline$(n-1,1)$ & 0 & 1 & 1 & 1 & 1 & 1 & 1 \\
\hline$(n-2,2)$ & 1 & 1 & 2 & 2 & 2 & 2 & 2 \\
\hline$(n-3,3)$ & 0 & 1 & 1 & 2 & 2 & 2 & 2 \\
\hline$(n-4,4)$ & & & 1 & 1 & 2 & 2 & 2 \\
\hline$(n-5,5)$ & & & & & 0 & 1 & 1 \\
\hline$(n-6,6)$ & & & & & & & 1 \\
\hline$(n-3,2,1)$ & 0 & 1 & 1 & 1 & 1 & 1 & 1 \\
\hline$(n-4,3,1)$ & & 0 & 1 & 1 & 1 & 1 & 1 \\
\hline$(n-5,4,1)$ & & & & 1 & 1 & 1 & 1 \\
\hline$(n-4,2,2)$ & 1 & 1 & 2 & 2 & 2 & 2 & 2 \\
\hline$(n-5,3,2)$ & & & 0 & 1 & 1 & 1 & 1 \\
\hline$(n-6,4,2)$ & & & & & 1 & 1 & 1 \\
\hline$(n-5,2,2,1)$ & & 1 & 1 & 1 & 1 & 1 & 1 \\
\hline$(n-6,2,2,2)$ & & & 1 & 1 & 1 & 1 & 1 \\
\hline$(n-4,1,1,1,1)$ & 1 & 1 & 1 & 1 & 1 & 1 & 1 \\
\hline$(n-5,2,1,1,1)$ & & 1 & 1 & 1 & 1 & 1 & 1 \\
\hline$(n-5,1,1,1,1,1)$ & 0 & 1 & 1 & 1 & 1 & 1 & 1 \\
\hline$(n-6,2,1,1,1,1)$ & & & 1 & 1 & 1 & 1 & 1 \\
\hline
\end{tabular}

TABLE 1

Our interest for this particular case arises from the context of Majorana theory (see [4]). More specifically, this result is needed for computing the linear span of $3 A$-axes in a standard Majorana representation of $S_{n}$ (see [3]).

\section{A REMARK ON PERMUTATiON MODULES}

Lemma 2.1. Let $H \leq N \leq N_{G}(H)$. With the notation established in Section 1, $C_{M_{H}^{G}}\left(N^{\tau}\right)$ is an $F[G]$-submodule of $M_{H}^{G}$ isomorphic to $M_{N}^{G}$.

Proof. Since the elements of $N^{\tau}$ are $F[G]$-automorphisms of $M_{H}^{G}$, we have that $C_{M_{H}^{G}}\left(N^{\tau}\right)$ is an $F[G]$-submodule of $M_{H}^{G}$. Let $\mathcal{G}$ (resp. $\mathcal{N}$ ) be a right transversal of $N$ in $G$ (resp. of $H$ in $N$ ), then $\{x g \mid x \in \mathcal{N}, g \in \mathcal{G}\}$ is a right transversal of $H$ in $G$ and, since $N^{\tau}$ is transitive on the set $\{H x g \mid x \in N\}$, the element

$$
\sum_{g \in \mathcal{G}} \sum_{x \in \mathcal{N}} a_{x, g} H x g \in M_{H}^{G}\left(\text { with } a_{x, g} \in F\right),
$$

centralizes $N^{\tau}$ if and only if, for every $g \in \mathcal{G}$, the coefficients $a_{x, g}$ have a constant value. Therefore the linearly independent elements $\sum_{x \in \mathcal{N}} H x g$, where $g \in \mathcal{G}$, generate $C_{M_{H}^{G}}\left(N^{\tau}\right)$ and the map

$$
\gamma: C_{M_{H}^{G}}\left(N^{\tau}\right) \rightarrow M_{N}^{G}
$$


defined by

$$
\sum_{g \in \mathcal{G}} a_{g} \sum_{x \in \mathcal{N}} H x g \mapsto \sum_{g \in \mathcal{G}} a_{g} N g
$$

is an $F[G]$-isomorphism.

Theorem 1.1 follows immediately from Lemma 2.1 and from the following generalisation of Lemma 4 in [3] (whose proof is essentially the same).

Lemma 2.2. Let $G$ and $K$ be finite groups, $M$ an $(F[G], F[K])$-bimodule, and $S$ an irreducible $F[G]$-module. Then the multiplicity of $S$ as a composition factor of the $F[G]$-module $C_{M}(K)$ is equal to the multiplicity of the trivial $F[K]$-module in $\operatorname{Hom}_{F[G]}(S, M)$.

Assume now that $G=A\langle\omega\rangle$, where $A$ is a normal subgroup and $\omega$ is an involution not contained in $A$. Let $Y$ be a subgroup of $G$ containing $\omega, N$ a subgroup of $G$ with $Y \leq N \leq N_{G}(Y)$, and set $H:=A \cap Y$. Then $H \leq N \leq N_{G}(H)$ and, via the action $\tau_{H}$ (resp. $\tau_{Y}$ ) of $N$ defined as in Section $1, M_{H}^{G}$ (resp. $M_{Y}^{G}$ ) is an $(F[G], F[N])$-bimodule. Let

$$
\operatorname{sgn}: G \rightarrow F
$$

be the linear character of $G$ whose value is 1 on the elements of $A$ and -1 otherwise. Let $U$ be the $(A, N \cap A)$-alternating $(F[G], F[N])$-bimodule, i.e. an $F$-vector space of dimension 1 on which $G$ (resp. $N$ ) acts with kernel $A$ (resp. $(N \cap A)$ ).

Lemma 2.3. $M_{H}^{G}$ is isomorphic, as an $(F[G], F[N])$-bimodule, to

$$
M_{Y}^{G} \oplus\left(M_{Y}^{G} \otimes_{F} U\right) .
$$

Proof. For $\pm \in\{+,-\}$, let $D_{ \pm}$be the $F[G]$-submodule of $M_{H}^{G}$ generated by the element $H \pm H \omega$. Let $\nu \in N$. Since $|\omega|=2,\langle H, \omega\rangle=Y$, and $Y$ is a normal subgroup of $N$, we have that $H \nu \omega=H \omega \nu$, whence

$$
(H \pm H \omega)^{\nu^{\tau}}=H \nu^{-1} \pm H \nu^{-1} \omega=H \nu^{-1} \pm H \omega \nu^{-1} \in D_{ \pm},
$$

showing that $D_{+}$and $D_{-}$are (also) $F[N]$-submodules. Let $\sigma_{1}, \ldots, \sigma_{t}$ a right transversal of $H$ in $A$. Then the $2 t$-tuple

$$
\mathcal{C}:=\left(H \sigma_{1}, \ldots, H \sigma_{t}, H \omega \sigma_{1}, \ldots, H \omega \sigma_{t}\right)
$$

is a basis of $M_{H}^{G}$ and

$$
\mathcal{C}_{ \pm}:=\left(H \sigma_{1} \pm H \omega \sigma_{1}, \ldots, H \sigma_{t} \pm H \omega \sigma_{t}\right)
$$

is a basis of $D_{ \pm}$. Since $G$ contains an involution, $\operatorname{char}(F) \neq 2$, whence

$$
M_{H}^{G}=D_{+} \oplus D_{-} .
$$

Fix $u_{0}$ a non zero vector in $U$ and let

$$
\theta_{+}: D_{+} \rightarrow M_{Y}^{G}\left(\text { resp. } \theta_{-}: D_{-} \rightarrow M_{Y}^{G} \otimes_{F} U\right)
$$

be the $F$-isomorphism defined, for every $i \in\{1, \ldots, t\}$, by

$$
\left.H \sigma_{i}+H \omega \sigma_{i} \mapsto Y \sigma_{i} \text { (resp. } H \sigma_{i}-H \omega \sigma_{i} \mapsto Y \sigma_{i} \otimes u_{0}\right) \text {. }
$$

Since $\theta_{+}$and $\theta_{-}$are isomomorphisms of $(F[G], F[N])$-bimodules, the result follows.

For an $F$-vector space $V$ denote by $V^{*}$ its dual space. 
Lemma 2.4. With the above notation, for every $F[G]$-module $S$, we have

$$
\operatorname{Hom}_{F[G]}\left(S, M_{Y}^{G} \otimes_{F} U\right) \cong_{F[N]} \operatorname{Hom}_{F[G]}\left(S \otimes U^{*}, M_{Y}^{G}\right) \otimes_{F} U .
$$

Proof. Set $M:=M_{Y}^{G}$. For $f \in S^{*}, m \in M$ and $u \in U$ let

$$
\begin{aligned}
& \phi_{f, m, u}: S \rightarrow M \otimes U \quad \text { and } \quad \bar{\phi}_{f, m, u}: S \otimes U^{*} \rightarrow M \\
& s \mapsto f(s) m \otimes u \quad \text { and } \quad s \otimes g \quad \mapsto \quad f(s) g(u) m .
\end{aligned}
$$

By [8, Chapter XVI, Propositions 1.1 and 1.2 and Corollaries 5.5 and 5.6] there is a unique (natural) isomorphism of $F$-spaces

$$
\zeta: \operatorname{Hom}_{F}\left(S, M \otimes_{F} U\right) \rightarrow \operatorname{Hom}_{F}\left(S \otimes_{F} U^{*}, M\right)
$$

such that, for every $f, m$, and $u$ as above,

$$
\phi_{f, m, u} \mapsto \bar{\phi}_{f, m, u}
$$

and, by [1, Proof of Theorem 43.14], $\zeta$ induces, by restriction, an $F$-space isomorphism $\zeta_{0}$ between $\operatorname{Hom}_{F[G]}\left(S, M \otimes_{F} U\right)$ and $\operatorname{Hom}_{F[G]}\left(S \otimes_{F} U^{*}, M\right)$. Let $\delta \in N$. Since $\left(\phi_{f, m, u}\right)^{\delta}=\phi_{f, m^{\delta}, u^{\delta}}$, for every $s \in S$ and $g \in U^{*}$, we have that

$$
\begin{aligned}
(s \otimes g)^{\left(\phi_{f, m, u}\right)^{\delta \zeta}} & =f(s) g\left(u^{\delta}\right) m^{\delta} \\
& =\operatorname{sgn} \delta f(s) g(u) m^{\delta} \\
& =\operatorname{sgn} \delta(s \otimes g)^{\left(\phi_{f, m, u}\right)^{\zeta \delta}},
\end{aligned}
$$

whence $\left(\phi_{f, m, u}\right)^{\delta \zeta}=\operatorname{sgn} \delta\left(\phi_{f, m, u}\right)^{\zeta \delta}$. Therefore, the required $F[N]$-isomorphism is obtained by fixing a generator $u_{0}$ of $U$ and composing $\zeta_{0}$ with the $F$-isomorphism

$$
\begin{aligned}
\operatorname{Hom}_{F}\left(S \otimes_{F} U^{*}, M\right) & \rightarrow \operatorname{Hom}_{F}\left(S \otimes_{F} U^{*}, M\right) \otimes_{F} U . \\
\phi & \mapsto \phi \otimes u_{0}
\end{aligned}
$$

Corollary 2.5. For every irreducible $F[G]$-module $S$, the multiplicity of the trivial $F[N]$-module in $\operatorname{Hom}_{F[G]}\left(S, M_{Y}^{G} \otimes_{F} U\right)$ is equal to the multiplicity of the Aalternating $F[N]$-module in $\operatorname{Hom}_{F[G]}\left(S \otimes U^{*}, M_{Y}^{G}\right)$.

Proposition 2.6. Let $A$ be a normal subgroup of $G$ and let $\omega \in G$ be an involution such that $G=A\langle\omega\rangle$. Let $Y$ and $N$ be subgroups of $G$ such that $\omega \in Y$ and $A \cap Y \leq N \leq N_{G}(Y)$. Then, for every irreducible $F[G]$-module $S$, the multiplicity of $S$ in $M_{N}^{G}$ is equal to the sum of the multiplicity of the trivial $F[N]$ module in $\operatorname{Hom}_{F[G]}\left(S, M_{Y}^{G}\right)$ with the multiplicity of the A-alternating $F[N]$-module in $\operatorname{Hom}_{F[G]}\left(S \otimes U^{*}, M_{Y}^{G}\right)$.

Proof. By Lemma 2.3 and Lemma 2.4, we have that

$$
\begin{aligned}
\operatorname{Hom}_{F[G]}\left(S, M_{Y \cap A}^{G}\right) & \cong_{F[N]} \operatorname{Hom}_{F[G]}\left(S, M_{Y}^{G}\right) \oplus \operatorname{Hom}_{F[G]}\left(S, M_{Y}^{G} \otimes_{F} U\right) \\
& \cong{ }_{F[N]} \operatorname{Hom}_{F[G]}\left(S, M_{Y}^{G}\right) \oplus\left(\operatorname{Hom}_{F[G]}\left(S \oplus U^{*}, M_{Y}^{G}\right) \otimes_{F} U\right),
\end{aligned}
$$

and the result follows by Lemma 2.1 . 


\section{Permutation modules for the symmetric Group}

From now on, assume $G$ is the symmetric group $S_{n}$ on the set $\{1, \ldots, n\}, A$ the alternating group $A_{n}$, and $F$ a field of characteristic 0 . Refer to [6] for basic facts about ordinary representation theory of the symmetric groups. For better readability, we briefly recall the notation of [3, Section 4$]$.

Let $\mu:=\left(\mu_{1}, \ldots, \mu_{r}\right)$ and $\lambda:=\left(\lambda_{1}, \ldots, \lambda_{s}\right)$ be partitions of $n$. We shall always assume that $\mu_{i} \geq \mu_{j}$ (resp. $\lambda_{i} \geq \lambda_{j}$ ), if $i<j$. Let $\mathcal{B}_{\mu}:=\left\{B_{1}, \ldots, B_{r}\right\}$, where $B_{r}:=\left\{1, \ldots, \mu_{r}\right\}$, and, for every $i \in\{1, \ldots, r-1\}$,

$$
B_{r-i}:=\left\{\sum_{j=0}^{i-1} \mu_{r-j}+1, \ldots, \sum_{j=0}^{i} \mu_{r-j}\right\} .
$$

The block normaliser $N_{\mu}$ is the subgroup of all elements of $S_{n}$ that induce a permutation on $\mathcal{B}_{\mu}$. The kernel of the action induced by $N_{\mu}$ on $\mathcal{B}_{\mu}$ is the Young subgroup $Y_{\mu}$ associated to $\mu$, defined by:

$$
Y_{\mu}:=S_{\left\{1, \ldots, \mu_{r}\right\}} \times S_{\left\{\mu_{r}+1, \ldots, \mu_{r}+\mu_{r-1}\right\}} \times \ldots \times S_{\left\{n-\mu_{1}+1, \ldots, n\right\}} .
$$

Denote by $[\lambda]$ the diagram associated to $\lambda$. Recall that a $\lambda$-tableau is a bijection $t:[\lambda] \rightarrow\{1, \ldots, n\}$. Denote it, as usual, by replacing each node of $[\lambda]$ by its image under $t$. $S_{n}$ acts on the set of all $\lambda$-tableaux by composition on the right: $t^{\sigma}:=t \sigma$, for every $\lambda$-tableaux $t$ and $\sigma \in S_{n}$. Given a $\lambda$-tableau $t$, the $\lambda$-tabloid $\{t\}$ associated to $t$ is the orbit of $t$ under the action of the rows stabiliser of $t$ in $S_{n}$. The action of $S_{n}$ on the $\lambda$-tableaux induces an action of $S_{n}$ on the set of all $\lambda$-tabloids. Let $M^{\lambda}$ be the permutation module of $S_{n}$ on the set of all $\lambda$-tabloids. Since the stabiliser of a $\lambda$-tabloid is conjugate in $S_{n}$ to $Y_{\lambda}, M^{\lambda}$ is $F\left[S_{n}\right]$-isomorphic to $M_{Y_{\lambda}}^{S_{n}}$. From now on, we fix a $\lambda$-tableau $t_{\lambda}$, so that the Specht module $S^{\lambda}$ is the cyclic $F\left[S_{n}\right]$-submodule of $M^{\lambda}$ generated by

$$
e_{t_{\lambda}}:=\sum_{\sigma \in C_{t_{\lambda}}}(\operatorname{sgn} \sigma)\left\{t_{\lambda}\right\}^{\sigma},
$$

where $C_{t_{\lambda}}$ is the columns stabiliser of $t_{\lambda}$. A $\lambda$-tableau $T$ of type $\mu$ is a function $T:[\lambda] \rightarrow\{1, \ldots, r\}$, such that $\left|T^{-1}(i)\right|=\mu_{i}$, for every $i \in\{1, \ldots, r\}$. $T$ is called semistandard if the entries are non-decreasing along the rows of $T$ and strictly increasing down the columns of $T$. Denote with $\mathcal{F}(\lambda, \mu)$ the set of all $\lambda$-tableaux of type $\mu$ and by $\mathcal{F}_{0}(\lambda, \mu)$ the set of all semistandard $\lambda$-tableaux of type $\mu$. Define an action (depending on $t_{\lambda}$ ) of the symmetric group $S_{n}$ on $\mathcal{F}(\lambda, \mu)$ setting, for every $T \in \mathcal{F}(\lambda, \mu)$ and every $\sigma \in S_{n}$,

$$
T^{\sigma}:=t_{\lambda} \sigma^{-1} t_{\lambda}^{-1} T
$$

This action is equivalent to the action of $S_{n}$ on the set of $\mu$-tabloids (see [6, p. 44]), so that the module $M^{\mu}$ is isomorphic to the permutation module $M^{\lambda \mu}$ associated to the above action of $S_{n}$ on the set $\mathcal{F}(\lambda, \mu)$.

Let $N_{S_{r}}(\mu)$ be the normaliser of the partition $\mu$, that is the subgroup of $S_{r}$ containing all the elements $\delta \in S_{r}$ such that $\mu_{i^{\delta}}=\mu_{i}$ for every $i \in\{1, \ldots, r\}$, and let

$$
\pi_{\mu}: N_{\mu} \rightarrow N_{S_{r}}(\mu)
$$

be the map sending $\sigma \in S_{n}$ to the permutation $\bar{\sigma} \in S_{r}$ defined, for every $i \in$ $\{1, \ldots, r\}$, by the condition $i^{\bar{\sigma}}=j \Longleftrightarrow\left(B_{i}\right)^{\sigma}=B_{j}$. Then $\pi_{\mu}$ is a well defined surjective group homomorphism with kernel $Y_{\mu}$, whence $N_{S_{r}}(\mu) \cong N_{\mu} / Y_{\mu}$. 
For every $T \in \mathcal{F}(\lambda, \mu)$ and $\delta \in N_{S_{r}}(\mu)$, let

$$
T^{\delta}:=T \delta
$$

so that $T^{\delta}$ is the $\lambda$-tableau of type $\mu$ obtained from $T$ by replacing every node $x$ of $T$ by $x^{\delta}$, and let $\alpha_{\delta}: M^{\lambda \mu} \rightarrow M^{\lambda \mu}$ be the $F$-automorphism induced on $M^{\lambda \mu}$ by the permutation $T \mapsto T^{\delta}$ of its basis $\mathcal{F}(\lambda, \mu)$.

Lemma 3.1. With the above notation, $\alpha_{\delta}$ is an $F\left[S_{n}\right]$-automorphism of $M^{\lambda \mu}$ and the map

$$
\begin{aligned}
\alpha: N_{S_{r}}(\mu) & \rightarrow \operatorname{Aut}_{F\left[S_{n}\right]}\left(M^{\lambda \mu}\right) \\
\delta & \mapsto \alpha_{\delta}
\end{aligned}
$$

is a group homomorphism, thus defining a structure of $\left(F\left[S_{n}\right], F\left[N_{S_{r}}(\mu)\right]\right)$-bimodule on $M^{\lambda \mu}$ and $M^{\mu}\left(\cong_{F\left[S_{n}\right]} M^{\lambda \mu}\right)$.

Proof. This follows immediately from the definitions (note that the action of $N_{S_{r}}(\mu)$ on $M^{\mu}$ permutes the rows of equal length of the $\mu$-polytabloids).

Let $T_{0}$ be the $\lambda$-tableau of type $\mu$ defined, for each $(h, k) \in[\lambda]$, by the condition:

$$
(h, k)^{T_{0}}=i \Longleftrightarrow(h, k)^{t_{\lambda}} \in B_{i} .
$$

Lemma 3.2. With the above notation, $Y_{\mu}$ is the stabiliser of $T_{0}$ in $S_{n}$ and there is an $F\left[S_{n}\right]$-isomorphism (of cyclic $F\left[S_{n}\right]$-modules)

$$
\xi: M_{Y_{\mu}}^{S_{n}} \rightarrow M^{\lambda \mu}
$$

such that $\left(Y_{\mu}\right)^{\xi}=T_{0}$.

Proof. This follows from the definition of $T_{0}$, since the action of $S_{n}$ on the $S_{n}$-orbit of $T_{0}$ is equivalent to the action on the set of right cosets of $Y_{\mu}$ in $S_{n}$.

Given a bijection $f: X \rightarrow Z$, between two sets $X$ and $Z$, denote by $f^{*}$ the map

$$
\begin{aligned}
f^{*}: S_{X} & \rightarrow S_{Z} \\
\phi & \mapsto f^{-1} \phi f
\end{aligned}
$$

Lemma 3.3. Let $\lambda$ and $\mu$ be partitions of $n$ and $\tau: N_{\mu} \rightarrow \operatorname{Aut}_{F\left[S_{n}\right]}\left(M^{\mu}\right)$ be the map defined in Section 1. Then the following diagram commutes.

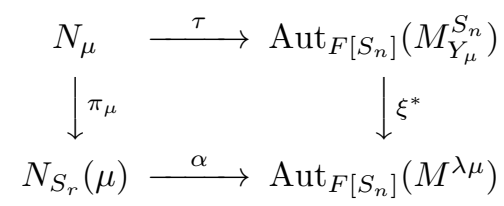

Proof. By definition we have that, for every $\delta \in N_{\mu}$,

$$
\delta t_{\lambda}^{-1} T_{0}=t_{\lambda}^{-1} T_{0} \delta^{\pi_{\mu}} .
$$

Thus for every $x \in N_{\mu}$, we have

$$
\left(T_{0}\right)^{x^{\tau \xi^{*}}}=\left(T_{0}\right)^{x^{-1}}=t_{\lambda} x t_{\lambda}^{-1} T_{0}=t_{\lambda} t_{\lambda}^{-1} T_{0} x^{\pi_{\mu}}=\left(T_{0}\right)^{x^{\pi_{\mu} \alpha}}
$$

and the result follows, since $M^{\lambda \mu}$ is a cyclic $F\left[S_{n}\right]$-module generated by $T_{0}$.

Using Lemma 3.3 we can restate Theorem 1.1 and Proposition 2.6 when the group $G$ is the symmetric group. Set $H_{\lambda \mu}:=\operatorname{Hom}_{F\left[S_{n}\right]}\left(S^{\lambda}, M^{\mu}\right)$. 
Theorem 3.4. With the above notation, let $Y_{\mu} \leq N \leq N_{\mu}$. Then, for every partition $\lambda$ of $n$, the multiplicity of $S^{\lambda}$ as a composition factor of $M_{N}^{S_{n}}$ is equal to the multiplicity of the trivial $F\left[N^{\pi_{\mu}}\right]$-module in $H_{\lambda \mu}$.

Proof. It follows from Lemma 3.3 and Theorem 1.1.

Denote by $\lambda^{\prime}$ the conjugate of $\lambda$, that is the partition whose diagram $\left[\lambda^{\prime}\right]$ is the transposed of $[\lambda]$. Write $\lambda \unrhd \mu$ if and only if $s \leq r$ and $\sum_{i=1}^{k} \lambda_{i} \geq \sum_{i=1}^{k} \mu_{i}$ for every $k \in\{1, \ldots, s\}$ and let $\Lambda_{\mu}$ be the set of partitions $\lambda$ such that $\lambda \unrhd \mu$.

Proposition 3.5. Let $\mu$ be a partition of $n, \mu \neq\left(1^{n}\right)$ and let $N \leq N_{\mu}$ such that $N \cap Y_{\mu}=1$ and for every $x \in N$, $\operatorname{sgn} x^{\pi_{\mu}}=\operatorname{sgn} x$. Then, the $F\left[S_{n}\right]$-module $M_{N\left(Y_{\mu} \cap A_{n}\right)}^{S_{n}}$ decomposes as a direct sum of irreducible submodules as follows:

$$
M=\bigoplus_{\lambda \in \Lambda_{\mu}}\left(m_{\lambda} S^{\lambda} \oplus n_{\lambda} S^{\lambda^{\prime}}\right)
$$

where $m_{\lambda}$ is equal to the multiplicity of the trivial $F\left[N^{\pi_{\mu}}\right]$-module in $H_{\lambda \mu}$, and $n_{\lambda}$ is equal to the multiplicity of the $A_{r}$-alternating $F\left[N^{\pi_{\mu}}\right]$-module of $H_{\lambda \mu}$.

Proof. Let $\lambda$ be a partition of $n$ and let $U$ be the $\left(A_{n}, A_{r}\right)$-alternating $\left(F\left[S_{n}\right], F\left[N_{S_{r}}(\mu)\right]\right)$ bimodule. Then $U$ is an $F[N]$-module via $\pi_{\mu}$, and, since by hypothesis $\operatorname{sgn} x^{\pi_{\mu}}=$ $\operatorname{sgn} x$ for every $x \in N, U$ is the alternating $F[N]$-module. By Theorem $[6$, Theorem 6.7] (that holds for any field of characteristic 0 ),

$$
S^{\lambda} \otimes_{F} U^{*} \cong_{F\left[S_{n}\right]} S^{\lambda} \otimes_{F} U \cong_{F\left[S_{n}\right]} S^{\lambda^{\prime}} .
$$

By Proposition 2.6 (with $A=A_{n}, Y=Y_{\mu}$, and $\omega=(n, n-1)$ ) and Lemma 3.3 we have that the multiplicity of $S^{\lambda}$ in $M_{N\left(Y_{\mu} \cap A_{n}\right)}^{S_{n}}$ is given by the sum of the multiplicity of the trivial $F\left[N^{\pi_{\mu}}\right]$-module in $\operatorname{Hom}_{F\left[S_{n}\right]}\left(S^{\lambda}, M^{\mu}\right)$ with the multiplicity of the alternating $F\left[N^{\pi_{\mu}}\right]$-module in $\operatorname{Hom}_{F\left[S_{n}\right]}\left(S^{\lambda^{\prime}}, M^{\mu}\right)$, and the result follows since, by [6, Corollary 13.17], $\operatorname{Hom}_{F\left[S_{n}\right]}\left(S^{\lambda}, M^{\mu}\right)=0$ unless $\lambda \unrhd \mu$.

\section{Action of $N_{S_{r}}(\mu)$ ON $H_{\lambda \mu}$}

By Theorem 3.4 (resp. Proposition 3.5), we are reduced to compute the dimension of the trivial (resp. trivial and alternating) $F\left[N^{\rho_{\mu}}\right]$-module of $H_{\lambda \mu}$. We do so by explicitly computing the matrices associated to the elements of a generating set of $N^{\rho_{\mu}}$, with respect to a basis $\mathcal{B}_{\lambda \mu}$ of $H_{\lambda \mu}$. For every $T \in \mathcal{F}(\lambda, \mu)$, set

$$
\mathcal{R}(T):=\left\{T^{\delta} \mid \delta \in R_{t_{\lambda}}\right\},
$$

where $R_{t_{\lambda}}$ is the row stabiliser of $t_{\lambda}$ in $S_{n}$. Since $S^{\lambda}$ is a cyclic $F\left[S_{n}\right]$-module generated by $e_{t_{\lambda}}$, for every $T \in \mathcal{F}(\lambda, \mu)$ there is a unique element $\hat{\theta}_{T}$ in $H_{\lambda \mu}$ such that

$$
e_{t_{\lambda}}^{\hat{\theta}_{T}}=\sum_{\sigma \in C_{t_{\lambda}}} \operatorname{sgn} \sigma \sum_{S \in \mathcal{R}(T)} S^{\sigma}
$$

Let

$$
\mathcal{B}_{\lambda \mu}:=\left(\left\{\hat{\theta}_{T} \mid T \in \mathcal{F}_{0}(\lambda, \mu)\right\}, \leq_{\lambda \mu}\right),
$$

where $\leq_{\lambda \mu}$ is the order induced by the following "lexicographic" order on $\mathcal{F}_{0}(\lambda, \mu)$ :

$$
\hat{\theta}_{R} \leq_{\lambda \mu} \hat{\theta}_{S} \text { if and only if } \sum_{(i, j) \in[\lambda]} n^{n^{i}+j}(i, j)^{R} \geq \sum_{(i, j) \in[\lambda]} n^{n^{i}+j}(i, j)^{S} .
$$


By [6, Theorem 13.13], $\mathcal{B}_{\lambda \mu}$ is a basis for $H_{\lambda \mu}$, thus, for every $\theta \in H_{\lambda \mu}$, there exist unique scalars $c_{\theta, S}$, where $S$ ranges in $\mathcal{F}_{0}(\lambda, \mu)$, such that

$$
\theta=\sum_{S \in \mathcal{F}_{0}(\lambda, \mu)} c_{\theta, S} \hat{\theta}_{S}
$$

Since $\mathcal{F}(\lambda, \mu)$ is a linearly independent set of generators of $M^{\lambda \mu}$, for every $\theta \in H_{\lambda \mu}$, there exist unique scalars $d_{\theta, R}$, where $R$ ranges in $\mathcal{F}(\lambda, \mu)$, such that

$$
e_{t_{\lambda}}^{\theta}=\sum_{R \in \mathcal{F}(\lambda, \mu)} d_{\theta, R} R
$$

For $\theta$ as above, let

$$
s(\theta):=\sum_{R \in \mathcal{F}_{0}(\lambda, \mu)} d_{\theta, R} R
$$

be the semistandard part of $e_{t_{\lambda}}^{\theta}$.

Note that, in general, for $S \in \mathcal{F}_{0}(\lambda, \mu)$, the coefficients $c_{\theta, S}$ 's do not coincide with the $d_{\theta, S}$ 's: consider, e.g., the following $(2,2,1)$-tableaux of type $\left(1^{5}\right)$ :

$$
T:=\begin{aligned}
& 14 \\
& 25 \\
& 3
\end{aligned}
$$

If we compute $e_{t_{\lambda}}^{\hat{\theta}_{T}}$, we see that, in the summands of the righthandside of Equation (4.4), together with $T$, the following "parvenu" semistandard $\lambda$-tableaux of type $\mu$ appears too:

$$
T^{\delta \sigma}=\begin{aligned}
& 12 \\
& 34 \\
& 5
\end{aligned},
$$

where $\delta$ is the rows stabilising permutation interchanging the two entries in the second row of $T$ and $\sigma$ is the column stabilising permutation interchanging the last two entries of the first column and the two entries of the second column of $T^{\delta}$. In order to deal with such parvenus, given $T \in \mathcal{F}(\lambda, \mu)$, set

$$
b_{i}(T):=\sum_{j=1}^{\lambda_{i}} j(i, j)^{T} \text { for every } i \in\{1, \ldots, s\}
$$

and

$$
b(T):=\sum_{(i, j) \in[\lambda]} j(i, j)^{T}=\sum_{i=1}^{s} b_{i}(T) .
$$

Thinking of the entries of $T$ as weights, $b_{i}(T)$ (resp. $b(T)$ ) can be viewed as the (non normalized) horizontal coordinate of the center of mass of the $i$-th row of $T$ (resp. of $T$ itself). The following lemma shows that this coordinate is maximal precisely when the heaviest weights are placed as far right as possible.

Lemma 4.1. With the above notations,

(1) for every $\sigma \in C_{t_{\lambda}}$, we have that $b(T)=b\left(T^{\sigma}\right)$;

(2) if $(i, j)^{T} \leq(i, h)^{T}$ for every $1 \leq j \leq h \leq \lambda_{i}$, then, for every $\sigma$ in $R_{t_{\lambda}}$, we have that $b_{i}\left(T^{\sigma}\right) \leq b_{i}(T)$, with equality if and only if $(i, j)^{T^{\sigma}}=(i, j)^{T}$ for every $1 \leq j \leq \lambda_{i}$;

(3) if $T$ is semistandard, then, for every $\delta \in R_{t_{\lambda}}$, either $T=T^{\delta}$ or $b(T)>b\left(T^{\delta}\right)$.

Proof. (1) follows immediately from the definitions of $b_{i}(T)$ and $b(T)$, (2) is an elementary exercise and (3) follows from (2). 
Lemma 4.2. Let $\theta$ and $\zeta$ be in $H_{\lambda \mu}$, then $\theta=\zeta$ if and only if $s(\theta)=s(\zeta)$.

Proof. It is enough to show that $\theta=0$ if and only if $s(\theta)=0$. Since $S^{\lambda}$ is a cyclic $F\left[S_{n}\right]$-module generated by $e_{t_{\lambda}}$, we have that $\theta=0$ implies $e_{t_{\lambda}}^{\theta}=0$ and hence $s(\theta)=0$. Conversely, assume $\theta \neq 0$. Let $S \in \mathcal{F}_{0}(\lambda, \mu)$, with $c_{\theta, S} \neq 0$ and $b(S)$ as large as possible. Then, by the definition of the $\hat{\theta}_{T}$ 's and by Lemma 4.1, it follows that $d_{\theta, S}=c_{\theta, S} \neq 0$.

For $R \in \mathcal{F}(\lambda, \mu)$, let $\Gamma(R)$ be the quiver with vertex set

$$
V(R):=\mathcal{F}_{0}(\lambda, \mu) \cup\{R\}
$$

and edge set

$$
E(R):=\left\{(\sigma, S, T) \mid \sigma \in C_{t_{\lambda}}, S \in V(R), T \in V(R) \backslash\{R, S\} \text { and } T^{\sigma^{-1}} \in \mathcal{R}(S)\right\},
$$

where, for each edge $(\sigma, S, T), S$ is the source and $T$ is the target. Given a vertex $T \in V(R)$, let $\mathcal{P}(T)$ (respectively $\mathcal{P}_{1}(T)$ ) be the set of paths (resp. paths of length 1 ) starting from $T$. Define a labeling on $E(R)$ by

$$
\chi: \begin{array}{ccc}
E(R) & \rightarrow\{-1,1\} \\
(\sigma, S, T) & \mapsto \operatorname{sgn} \sigma
\end{array} .
$$

Given a path $p:=\left(e_{1}, e_{2}, \ldots, e_{k}\right)$ in $\Gamma(R)$, with $e_{i}:=\left(\sigma_{i}, S_{i}, T_{i}\right)$ for $i \in\{1, \ldots, k\}$, denote by $l(p)$ the length $k$ of $p$, by $S_{1}^{p}$ the ending vertex $T_{k}$ of $p$, and set

$$
\chi(p):=\prod_{i=1}^{k} \chi\left(e_{i}\right) .
$$

Lemma 4.3. Let $\Gamma(R)$ be as above, then $\Gamma(R)$ contains no cycles.

Proof. By Lemma 4.1, for every $(\sigma, S, T) \in E(R)$ with $S \neq R$, we have $b(T)<b(S)$. And the claim follows since, by definition, $R$ is not a target.

Lemma 4.4. Let $\lambda$ and $\mu$ be partitions of $n$, then, for every $T \in \mathcal{F}(\lambda, \mu) \backslash \mathcal{F}_{0}(\lambda, \mu)$ and $T_{0} \in \mathcal{F}_{0}(\lambda, \mu)$ we have

$$
s\left(\hat{\theta}_{T}\right)=\sum_{p \in \mathcal{P}_{1}(T)} \chi(p) T^{p} \text { and } s\left(\hat{\theta}_{T_{0}}\right)=T_{0}+\sum_{p \in \mathcal{P}_{1}\left(T_{0}\right)} \chi(p) T_{0}^{p} .
$$

Proof. This follows immediately from the definitions.

Proposition 4.5. Let $\lambda$ and $\mu$ be partitions of $n$, then, for every $T \in \mathcal{F}(\lambda, \mu) \backslash$ $\mathcal{F}_{0}(\lambda, \mu)$, we have

$$
\hat{\theta}_{T}=\sum_{p \in \mathcal{P}(T)}(-1)^{l(p)+1} \chi(p) \hat{\theta}_{T^{p}} .
$$

Proof. Set $\theta:=\hat{\theta}_{T}+\sum_{p \in \mathcal{P}(T)}(-1)^{l(p)} \chi(p) \hat{\theta}_{T^{p}}$. By Lemma 4.2, it is sufficient to prove that $s(\theta)=0$. By Lemma 4.4, we have

$$
\begin{aligned}
s(\theta) & =s\left(\hat{\theta}_{T}\right)+\sum_{p \in \mathcal{P}(T)}(-1)^{l(p)} \chi(p) s\left(\hat{\theta}_{T^{p}}\right) \\
& =\sum_{p \in \mathcal{P}_{1}(T)} \chi(p) T^{p}+\sum_{p \in \mathcal{P}(T)}(-1)^{l(p)} \chi(p)\left(T^{p}+\sum_{q \in \mathcal{P}_{1}\left(T^{p}\right)} \chi(q)\left(T^{p}\right)^{q}\right) \\
& =\sum_{p \in \mathcal{P}(T)}(-1)^{l(p)} \chi(p) T^{p}-\sum_{p \in \mathcal{P}(T)}(-1)^{l(p)} \chi(p) T^{p}=0 .
\end{aligned}
$$


Corollary 4.6. Let Let $\lambda$ and $\mu$ be partitions of $n, T \in \mathcal{F}_{0}(\lambda, \mu)$ and $\varepsilon \in N_{S_{r}}(\mu)$. Then either $T^{\varepsilon} \in \mathcal{R}(S)$, for some $S \in \mathcal{F}_{0}(\lambda, \mu)$, in which case $\left(\hat{\theta}_{T}\right)^{\varepsilon}=\hat{\theta}_{S}$, or

$$
\left(\hat{\theta}_{T}\right)^{\varepsilon}=\sum_{p \in \mathcal{P}\left(T^{\varepsilon}\right)}(-1)^{l(p)+1} \chi(p) \hat{\theta}_{\left(T^{\varepsilon}\right)^{p}}
$$

Proof. This follows from [3, Lemma 5] and Proposition 4.5.

As an example consider the case

$$
\lambda=(5,5,1), \quad \mu=(5,1,1,1,1,1,1), \text { and } \varepsilon=(2,5,3,6,4,7) .
$$

Then $\mathcal{B}_{\lambda \mu}=\left(\hat{\theta}_{T_{1}}, \hat{\theta}_{T_{2}}, \hat{\theta}_{T_{3}}, \hat{\theta}_{T_{4}}, \hat{\theta}_{T_{5}}\right)$, where

$$
T_{1}=\begin{aligned}
& 11111 \\
& 23456 \\
& 7
\end{aligned}, T_{2}=\begin{aligned}
& 11111 \\
& 23457 \\
& 6
\end{aligned}, T_{3}=\begin{aligned}
& 11111 \\
& 23467 \\
& 5
\end{aligned}, T_{4}=\begin{aligned}
& 11111 \\
& 23567 \\
& 4
\end{aligned}, T_{5}=\begin{aligned}
& 11111 \\
& 24567 \\
& 3
\end{aligned},
$$

and

$$
T_{1}^{\varepsilon}=\begin{aligned}
& 11111 \\
& 56734
\end{aligned} .
$$

Since any of the $T_{i}$ 's, for $1 \leq i \leq 5$ can be obtained from $T_{1}^{\varepsilon}$ by a permutation of the second row followed by the transposition that interchanges the last two entries of the first column while, for every $\delta \in R_{t_{(5,5,1)}}, \sigma \in R_{t_{(5,5,1)}}$, and $\{i, j\} \subseteq\{1, \ldots, 5\}$, we have $T_{i}^{\delta \sigma}=T_{j}$ if and only if $i=j$, it follows that the quiver $\Gamma\left(T_{1}^{\varepsilon}\right)$ is

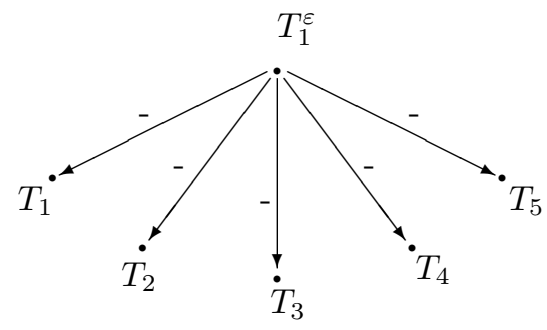

and by Corollary 4.6, $\left(\hat{\theta}_{T_{1}}\right)^{\varepsilon}=-\hat{\theta}_{T_{1}}-\hat{\theta}_{T_{2}}-\hat{\theta}_{T_{3}}-\hat{\theta}_{T_{4}}-\hat{\theta}_{T_{5}}$.

\section{Proof of Theorem 1.2}

Let $K_{n}$ be the normalizer in $S_{n}$ of the subgroup $\langle(1,2,3)(4,5,6)\rangle$. Then

$$
K_{n}=\langle(1,2,3),(4,5,6),(1,4)(2,5)(3,6),(2,3)(5,6)\rangle \times L_{n}
$$

where $L_{n} \cong S_{n-6}$. Consider first the case $n=6$ and set $\mu:=(3,3)$. We have that

$$
K_{6}=\left(Y_{(3,3)} \cap A_{6}\right) \times N \leq N_{(3,3)} \text {, where } N=\langle(1,4)(2,5)(3,6)\rangle .
$$

Then, $c:=(1,4)(2,5)(3,6)^{\pi_{\mu}}=(1,2)$, hence, for every element $x \in N$, we have that $\operatorname{sgn} x=\operatorname{sgn} x^{\pi_{\mu}}$. Since $\Lambda_{(3,3)}=\{(6),(5,1),(4,2),(3,3)\}$, by Proposition 3.5 
$M_{K_{6}}^{S_{6}}$ decomposes into $F\left[S_{6}\right]$-irreducible submodules as follows

$$
\begin{aligned}
M_{K_{6}}^{S_{6} \cong} & m_{(6)} S^{(6)} \oplus m_{(5,1)} S^{(5,1)} \oplus m_{(4,2)} S^{(4,2)} \oplus m_{(3,3)} S^{(3,3)} \oplus \\
& n_{(3,3)} S^{(2,2,2)} \oplus n_{(4,2)} S^{(2,2,1,1)} \oplus n_{(5,1)} S^{(2,1,1,1,1)} \oplus n_{(6)} S^{\left(1^{6}\right)},
\end{aligned}
$$

where for $\lambda \in \Lambda_{(3,3)}, m_{\lambda}$ is equal to the multiplicity of the trivial $F[\langle c\rangle]$-module in $H_{\lambda \mu}$ and $n_{\lambda}$ is equal to the multiplicity of the alternating $F[\langle c\rangle]$-module in $H_{\lambda \mu}$. By [6, Corollary 13.14], for every $\lambda \in \Lambda_{(3,3)}, H_{\lambda \mu}$ has dimension 1 and, by Corollary 4.6 and [6, Theorem 6.7], $c$ acts trivially on it, if $\lambda \in\{(6),(4,2)\}$, and as multiplication by -1 , if $\lambda \in\{(5,1),(3,3)\}$. Thus $m_{(6)}=m_{(4,2)}=n_{(5,1)}=n_{(3,3)}=1$ and $m_{(5,1)}=m_{(3,3)}=n_{(6)}=n_{(4,2)}=0$ as in the first column of Table 1. To get the second column, note that $K_{7}=K_{6} \leq S_{6}$, whence $M_{K_{7}}^{S_{7}}=M_{K_{6}}^{S_{6}} \uparrow S^{7}$, and the result follows by the Branching Theorem ([6, Theorem 9.2]).

Assume now $n \geq 8$ and let $\mu:=(n-6,1,1,1,1,1,1)$. Then $Y_{\mu} \leq K_{n} \leq N_{\mu}$ and

$$
K_{n}^{\pi_{\mu}}=\langle(2,5,3,6,4,7),(3,4)(5,6)\rangle .
$$

Set $a:=(2,5,3,6,4,7)$ and $b:=(3,4)(5,6)$. By [6, Corollary 13.17], the irreducible $F\left[S_{n}\right]$-submodules of $M^{\mu}$ are isomorphic to the Specht modules $S^{\lambda}$, where $\lambda$ belongs to the set

$$
\begin{aligned}
\Lambda_{(n-6,1,1,1,1,1,1)}= & \{(n),(n-1,1),(n-2,2),(n-3,3),(n-4,4),(n-5,5), \\
& (n-6,6),(n-2,1,1),(n-3,2,1),(n-4,3,1),(n-5,4,1), \\
& (n-6,5,1),(n-4,2,2),(n-5,3,2),(n-6,4,2),(n-6,3,3), \\
& (n-3,1,1),(n-4,2,1,1),(n-5,3,1,1),(n-6,4,1,1), \\
& (n-5,2,2,1),(n-6,3,2,1),(n-6,2,2,2),(n-4,1,1,1,1), \\
& (n-5,2,1,1,1),(n-6,3,1,1,1),(n-6,2,2,1,1), \\
& (n-5,1,1,1,1,1),(n-6,2,1,1,1,1), \mu\} .
\end{aligned}
$$

By Theorem 3.4, we can obtain the multiplicity of the Specht module $S^{\lambda}$ in $M_{K_{n}}^{S_{n}}$ once we determine, for every $\lambda \in \Lambda_{(n-6,1,1,1,1,1,1)}$, the multiplicity of the trivial $F[\langle a, b\rangle]$-module in $H_{\lambda \mu}$.

Lemma 5.1. For every $\lambda \in \Lambda_{(n-6,1,1,1,1,1,1)}$ and $n \geq 6+\lambda_{2}$, the multiplicity $m_{\lambda}$ of the Specht module $S^{\lambda}$ in $M_{K_{n}}^{S_{n}}$ does not depend on $n$.

Proof. The result follows since, for every $\lambda \in \Lambda_{(n-6,1,1,1,1,1,1)}$, the structure of the $F\left[N_{S_{r}}(\mu)\right]$-modules $H_{\lambda \mu}$ does not depend on $n$ when $n \geq 6+\lambda_{2}$.

Lemma 5.2. Let $0 \leq k \leq 6$ and $n \geq 6+k$. Then $m_{(n-k, k)}=2$, if $k \in\{2,3,4\}$, and $m_{(n-k, k)}=1$, if $k \in\{0,1,5,6\}$.

Proof. For $\lambda=(n-k, k)$, with $1 \leq k \leq 6$, we have that, by Corollary $4.6,\langle a, b\rangle$ permutes the elements of the basis $\mathcal{B}_{\lambda \mu}$ in the same way as it permutes the elements of $\mathcal{F}_{0}(\lambda, \mu)$, and this action is equivalent to the action of $\langle a, b\rangle$ on the set of $k$-subsets of $\{1, \ldots, 6\}$. Hence, the multiplicity of the trivial $F[\langle a, b\rangle]$-module in $H_{\lambda \mu}$ is equal to the number of $\langle a, b\rangle$-orbits on the set of $k$-subsets of $\{2, \ldots, 7\}$, and the result follows.

This gives the first seven rows of Table 1 with the exceptions of $m_{(5,3)}, m_{(4,4)}$, $m_{(5,4)}$, and $m_{(5,5)}$. From Lemma 5.1 and the case $n=7$, we have that, for every $n \geq 8$, the values of $m_{(n-4,1,1,1,1)}$ and $m_{(n-5,1,1,1,1,1)}$ are as given in Table 1 , while 
$m_{(n-3,1,1,1)}=m_{(n-6,1,1,1,1,1,1)}=m_{(n-2,1,1)}=0$. This completes the cases where $\lambda \in \Lambda_{(n-6,1,1,1,1,1,1)}$ with $\lambda_{2}=1$.

We consider now the case $m_{\lambda}$ where $\lambda=(n-6,5,1)$ and $n \geq 11$. By Lemma 5.1 we can reduce to $n=11$. Using Corollary 4.6 , as in the example at the end of that section (that, incidentally, gives the first row of the first matrix), one can show that the matrices associated to $a$ and $b$ with respect to the basis $\mathcal{B}_{\lambda \mu}$ are respectively

$$
\left(\begin{array}{rrrrr}
-1 & -1 & -1 & -1 & -1 \\
0 & 0 & 0 & 1 & 0 \\
0 & 0 & 0 & 0 & 1 \\
1 & 0 & 0 & 0 & 0 \\
0 & 1 & 0 & 0 & 0
\end{array}\right) \quad \text { and } \quad\left(\begin{array}{rrrrr}
1 & 0 & 0 & 0 & 0 \\
0 & 0 & 1 & 0 & 0 \\
0 & 1 & 0 & 0 & 0 \\
0 & 0 & 0 & 0 & 1 \\
0 & 0 & 0 & 1 & 0
\end{array}\right)
$$

whence, $m_{(5,5,1)}=0$. In a similar way one shows that $m_{(n-5,4,1)}=1, m_{(n-6,4,2)}=$ 1 , and $m_{(n-6,4,1,1)}=0$, for $n \geq 10$, and $m_{(5,5)}=0$. Note that, for $\lambda=(n-5,4,1)$, $H_{\lambda \mu}$ has dimension 24, while in the other cases the dimension is at most 10.

The remaining cases could be also dealt similarly, using Theorem 3.4 with $\mu=(n-6,1,1,1,1,1,1)$, but the dimensions involved increase (up to 45). To reduce the size of the matrices, it is more convenient to use Proposition 3.5 replacing $\mu$ with a different partition $\bar{\mu}$ as follows. Assume $n=8$. Set $J_{8}:=$ $N_{S_{8}}(\langle(3,4,5)(6,7,8)\rangle)$, and $\bar{\mu}:=(3,3,1,1)$. Then $J_{8}=\left(Y_{\bar{\mu}} \cap A_{8}\right) \times N \leq N_{\bar{\mu}}$, where $N=\langle(3,6)(4,7)(5,8),(1,2)\rangle$, and $N^{\pi_{\bar{\mu}}}=\langle c, d\rangle$, where $c:=(3,6)(4,7)(5,8)^{\pi_{\bar{\mu}}}$ and $d:=(1,2)^{\pi_{\bar{\mu}}}$. Then, for every $x \in N$, we have that $\operatorname{sgn} x=\operatorname{sgn} x^{\pi_{\bar{\mu}}}$. Since $\lambda_{2}=1$ if and only if $\lambda_{2}^{\prime}=1$,

$$
\begin{aligned}
\Lambda_{(3,3,1,1)}= & \{(8),(7,1),(6,1,1),(6,2),(5,1,1,1),(5,2,1),(5,3) \\
& (4,1,1,1,1),(4,2,1,1),(4,2,2),(4,3,1),(4,4),(3,2,2,1) \\
& (3,3,2),(3,3,1,1)\}
\end{aligned}
$$

and $(3,2,2,1)=(4,3,1)^{\prime}$, we are reduced to compute the multiplicities $m_{\lambda}$ and $n_{\lambda}$ for $\lambda$ in the set

$$
\{\{(6,2)(5,2,1),(5,3),(4,2,1,1),(4,3,1),(4,2,2),(4,4),(3,3,2),(3,3,1,1)\} .
$$

The matrices representing $c$ (resp. $d$ ), with respect to the basis $\mathcal{B}_{\lambda \bar{\mu}}$ defined as in Section 4, are given in the second (resp. third) column of Table 2. By Proposition 3.5, the dimension of their common 1-eigenspace (resp. -1-eigenspace) is $m_{\lambda}$ (resp. $n_{\lambda}$ ) and these are given in the fourth (resp. fith) column of Table 2. 


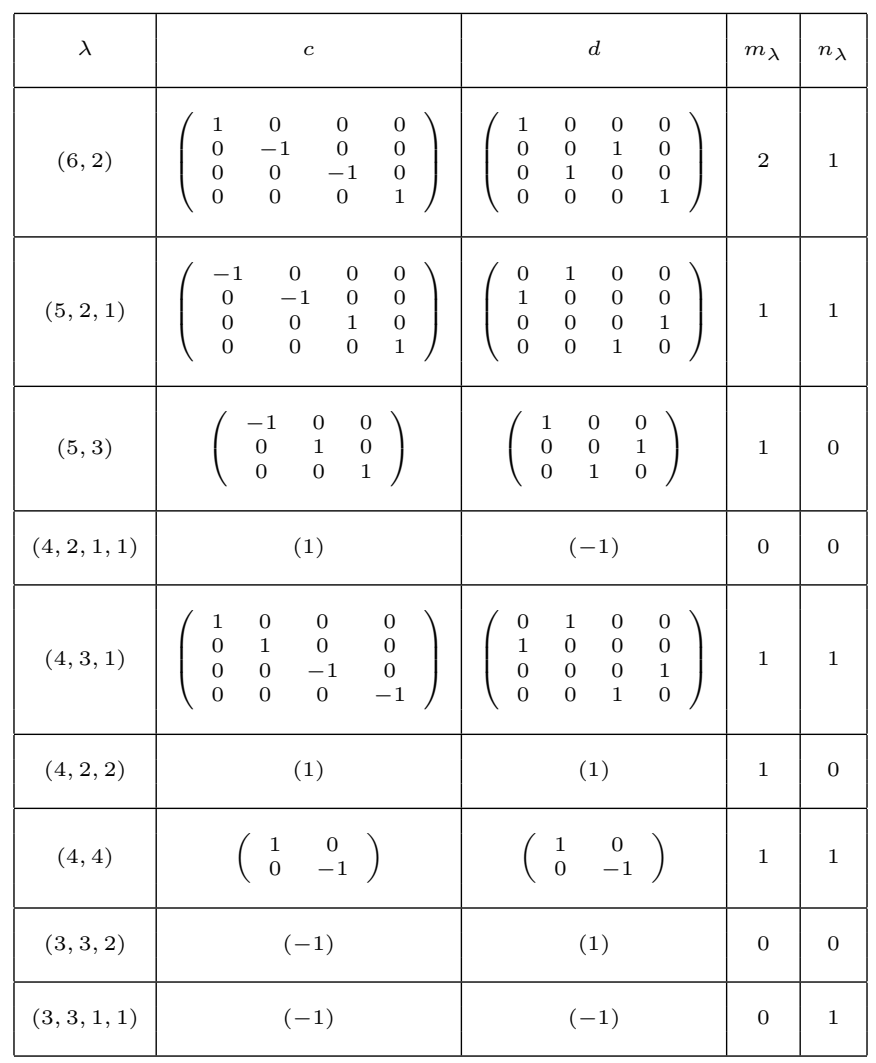

TABLE 2

By Proposition 3.5 and Lemma 5.1, this gives all the entries in the fourth column and in the rows corresponding to the partitions $(n-3,2,1),(n-5,2,2,1)(n-$ $6,2,2,2),(n-6,2,1,1,1,1),(n-5,2,1,1,1),(4,3,1)$, and $(n-4,2,2)$ of Table 1 , whilst showing that, for every $n \geq 8$, the values $m_{(n-4,2,1,1)}, m_{(n-6,2,2,1,1)}, m_{(3,3,2)}$, $m_{(3,3,1,1)}$, and $m_{(n-4,2,1,1)}$ are all 0 .

Similarly, for $n=9$, set $J_{9}:=N_{S_{9}}(\langle(4,5,6)(7,8,9)\rangle)$ and $\bar{\mu}:=(3,3,1,1,1)$. Then $J_{9}=\left(Y_{\bar{\mu}} \cap A_{9}\right) \times N \leq N_{\bar{\mu}}$, where $N=\langle(4,7)(5,8)(6,9),(1,2),(2,3)\rangle$ and $N^{\pi_{\bar{\mu}}}=\langle c, d, e\rangle$, where $c:=(4,7)(5,8)(6,9)^{\pi_{\bar{\mu}}}, d:=(1,2)^{\pi_{\bar{\mu}}}$ and $e:=(2,3)^{\pi_{\bar{\mu}}}$. Again, for every element $x \in N$, we have that $\operatorname{sgn} x=\operatorname{sgn} x^{\pi_{\bar{\mu}}}$, so Proposition 3.5 applies. Since

$$
\begin{aligned}
\Lambda_{(3,3,1,1,1)}= & \{(9),(8,1),(7,1,1),(7,2),(6,1,1,1),(6,2,1),(6,3),(5,1,1,1,1), \\
& (5,2,1,1),(5,2,2),(5,3,1),(5,4),(4,2,1,1,1),(4,2,2,1), \\
& (4,3,1,1),(4,3,2),(4,4,1),(3,3,3),(3,3,2,1),(3,3,1,1,1)\}
\end{aligned}
$$

and $(3,3,2,1)=(4,3,2)^{\prime}$, by Proposition 3.5, we need to determine the multiplicities $m_{\lambda}$ and $n_{\lambda}$ for

$$
\lambda \in\{(5,4),(5,3,1),(4,3,2),(3,3,3),(4,3,1,1),(3,3,1,1,1),(4,4,1)\} .
$$

As in the previous case we get that the multiplicities $m_{\lambda}$ and $n_{\lambda}$ are as in Table 3 : 


\begin{tabular}{|c|c|c|c|c|c|c|c|}
\hline$\lambda$ & $(5,4)$ & $(5,3,1)$ & $(4,3,2)$ & $(3,3,3)$ & $(4,3,1,1)$ & $(3,3,1,1,1)$ & $(4,4,1)$ \\
\hline$m_{\lambda}$ & 1 & 1 & 1 & 0 & 0 & 0 & 1 \\
\hline$n_{\lambda}$ & 0 & 0 & 0 & 0 & 1 & 1 & 1 \\
\hline
\end{tabular}

TABLE 3

\section{ACKNOWLEDGEMENTS}

The authors wish to thank Gunter Malle and Gabriel Navarro for helpful information about this topic.

\section{REFERENCES}

1. C. W. Curtis, I. Reiner, Representation Theory of Finite Groups and Associative Algebras. Chelsea Publishing, American Math. Soc., Providence, RI (1962)

2. W. Fulton, J. Harris, Representation theory, Graduate Texts in Mathematics 129, Springer, New York-Berlin-Heidelberg (1991)

3. C. Franchi, A. A. Ivanov, M. Mainardis, Standard Majorana representations of the symmetric groups, J. Algebraic Combin. .. (2016),

4. A. A. Ivanov, The Monster Group and Majorana Involutions, Cambridge Tracts in Mathematics 176. Cambridge University Press, Cambridge (2009).

5. N. Jacobson, Basic Algbera II. W. H. Freeman and Co., New York (1989)

6. James, G. D.: The Representation Theory of the Symmetric Groups. Lecture Notes in Mathematics 682, Springer, Berlin-Heidelberg (1978)

7. Isaacs, I. M.: Character Theory of finite Groups. Dover Publications Inc., New York (1994)

8. Lang, S.: Algebra. Graduate Texts in Mathematics 211, Springer, New York (2002)

Dipartimento di Matematica e Fisica, Università Cattolica del Sacro Cuore, Via Musei 41 I-25121 Brescia, ItAly

E-mail address: clara.franchi@unicatt.it

Department of Mathematics, Imperial College, 180 Qeen's Gt., London, SW7 2AZ, $\mathrm{UK}$

E-mail address: a.ivanov@imperial.ac.uk

Dipartimento di Scienze Matematiche, Informatiche e Fisiche, Universitì degli Studi Di Udine, Via Delle Scienze 206, I-33100 Udine, Italy

E-mail address: mario.mainardis@uniud.it 